\title{
ATIVIDADE ANTIMICROBIANA IN VITRO DO RIZOMA EM PÓ, DOS PIGMENTOS CURCUMINÓIDES E DOS ÓLEOS ESSENCIAIS DA Curcuma longaL.
}

\author{
In vitro antimicrobial activity of the ground rhizome, curcuminoid pigments and \\ essential oil of Curcuma longa $\mathbf{L}$.
}

\author{
Lúcia Péret-Almeida ${ }^{1}$, Cristina da Cunha Naghetini², Elzíria de Aguiar Nunan³ \\ Roberto Gonçalves Junqueira ${ }^{4}$, Maria Beatriz Abreu Glória ${ }^{5}$
}

\begin{abstract}
RESUMO
Este trabalho teve como objetivo avaliar a atividade antimicrobiana da cúrcuma em pó, da curcumina disponível no comércio, dos pigmentos curcuminóides purificados e dos óleos essenciais da cúrcuma. As amostras foram analisadas quanto aos teores de pigmentos curcuminóides por cromatografia líquida de alta eficiência. O óleo essencial foi também analisado quanto à densidade, índice de refração e perfil das substâncias voláteis por cromatografia gasosa e detector de ionização de chamas. A atividade antimicrobiana in vitro foi determinada pelo método de difusão em ágar com discos de papel estéreis, impregnados com os extratos, sendo os diâmetros dos halos de inibição medidos com paquímetro. Os extratos etanólicos da cúrcuma, da curcumina comercial, e dos pigmentos curcumina e desmetoxicurcumina não inibiram o crescimento de Staphylococcus aureus, Bacillus subtilis, Salmonella choleraesuis, Escherichia coli, Aspergillus niger, Saccharomyces cerevisiae, e Candida albicans. Apenas o extrato etanólico da bisdesmetoxicurcumina apresentou atividade antimicrobiana em relação ao B. subtilis. O óleo essencial da cúrcuma apresentou atividade antimicrobiana para o B. subtilis, $S$. choleraesuis, E. coli, A. niger e $S$. cerevisiae, sendo que essa atividade aumentou em função do aumento da concentração. Os halos de inibição, obtidos com o óleo essencial, foram significativos, quando comparados aos respectivos antibióticos tradicionais, cloranfenicol e anfotericina, indicando ser o óleo essencial da cúrcuma um agente antimicrobiano em potencial.
\end{abstract}

Termos para indexação: Atividade antimicrobiana, cúrcuma, pigmentos curcuminóides, óleo essencial, Curcuma longa.

\section{ABSTRACT}

The objective of this work was to investigate the antimicrobial activity of ground turmeric, commercially available curcumin, purified curcuminoid pigments and turmeric essential oils. The samples were analyzed for the levels of curcuminoid pigments by high performance liquid chromatography. The essential oil was also analyzed for density, refractive index and profile of volatile compounds by gas chromatography-flame ionization detection. The in vitro antimicrobial activity was determined by the agar diffusion method using sterile disks impregnated with the extracts and the diameters of the inhibition zones were measured using a caliper. The ethanolic extracts of turmeric, commercial curcumin, and the purified pigments curcumin and demethoxycurcumin did not inhibit the growth of Staphylococcus aureus, Bacillus subtilis, Salmonella choleraesuis, Escherichia coli, Aspergillus niger, Saccharomyces cerevisiae, and Candida albicans. Only the ethanolic extract of bisdemethoxycurcumin showed in vitro antimicrobial activity on $B$. subtilis. The essential oil of turmeric inhibited the growth of B. subtilis, S. choleraesuis, E. coli, A. niger and S. cerevisiae. The antimicrobial activity increased with an increase in the concentration of the essential oil. The inhibitory effects on the microorganisms were significant when compared to the respective traditional antibiotics chloranphenicol and anphotericin. This result indicates that the essential oil from turmeric is a potential antimicrobial agent.

Index terms: Antimicrobial activity, turmeric, curcuminoid pigments, essential oil, Curcuma longa.

(Recebido em 27 de abril de 2006 e aprovado em 14 de novembro de 2006)

\section{INTRODUÇÃO}

A deterioração tem sido uma das grandes preocupações e uma das maiores causas de perdas de alimentos, diminuição da produtividade e de danos à saúde do homem. O uso de aditivos tem sido efetivo na prevenção da deterioração de alimentos. Entretanto, existe uma tendência cada vez maior de utilizar aditivos naturais

\footnotetext{
${ }^{1}$ Farmacêutica, Doutora, Professora - Departamento de Alimentos/ALM - UNI-BH - Avenida Professor Mário Werneck, 1685, Estoril - 30455-610 - Belo Horizonte, MG - luciaperet@gmail.com

${ }^{2}$ Bióloga, Mestre - Departamento de Alimentos/ALM - Universidade Federal de Minas Gerais/UFMG - Avenida Antônio Carlos, 6627, Pampulha - 31270-901 Belo Horizonte, MG - kikanaghetini@ig.com.br

${ }^{3}$ Farmacêutica, Doutora, Professora Associado - Departamento de Produtos Farmacêuticos/PFA - Universidade Federal de Minas Gerais/UFMG Avenida Antônio Carlos, 6627, Pampulha - 31270-901 - Belo Horizonte, MG - enunan@farmacia.ufmg.br

${ }^{4}$ Farmacêutico, Doutor, Professor Associado - Departamento de Alimentos/ALM - Universidade Federal de Minas Gerais/UFMG - Avenida Antônio Carlos, 6627, Pampulha - 31270-901 - Belo Horizonte, MG - junkeira@netuno.ufmg.br

${ }^{5}$ Engenheira Alimentos, PhD, Professor Associado - Departamento de Alimentos/ALM - Universidade Federal de Minas Gerais/UFMG - Avenida Antônio Carlos, 6627, Pampulha -31270-901 - Belo Horizonte, MG - beatriz@farmacia.ufmg.br
} 
devido à conscientização sobre os efeitos tóxicos de alguns destes. Além disto, novos patógenos veiculados por alimentos têm surgido e tem-se observado o desenvolvimento de resistência de vários deles, aos biocidas usuais. Assim, pesquisas são necessárias para descobrir substâncias naturais capazes de substituir os aditivos tradicionais de maneira eficaz e segura (GUL et al., 2004; NEGI et al., 1999).

Várias plantas têm sido utilizadas no controle de agentes deteriorantes de alimentos por conterem substâncias antimicrobianas e por não causarem efeitos adversos ao homem. O rizoma da cúrcuma (Curcuma longa L.) tem sido muito utilizado na indústria alimentícia, como corante, aromatizante e como ingrediente no preparo de temperos, laticínios e pratos prontos (APISARIYAKUL et al., 1995; GOVINDARAJAN, 1980; SILVA et al., 2005). De acordo com Gul et al. (2004), a cúrcuma inibiu de forma significativa o crescimento in vitro de algumas bactérias, como o Staphylococcus aureus, no entanto, não inibiu Escherichia coli. Uma fraca atividade antibacteriana foi verificada por Singh et al. (2002) e Tönnesen \& Karlsen (1987) para a cúrcuma em relação a $S$. aureus, E. coli, Pseudomonas aeruginosa, S. epidermidis, e Salmonella typhimurium. De acordo com Kim et al. (2003), extratos da cúrcuma apresentaram atividade antifúngica in vitro para algumas cepas de Trichophyton e para Botrytis, Erysiphe, Phytophthora, Puccinia, Pyricularia e Rhizoctonia.

Baseado nestes estudos pode-se afirmar a atividade antimicrobiana da cúrcuma. Essa atividade tem sido associada aos pigmentos curcuminóides (Figura 1); entretanto a cúrcuma em pó contém, além dos pigmentos, os óleos essenciais. De acordo com Negi et al. (1999) e Singh et al. (2002), o óleo essencial da cúrcuma também apresenta atividade antimicrobiana.
Neste trabalho, determinou-se entre os componentes da cúrcuma, aqueles com potencial antimicrobiano. Investigou-se a atividade inibitória in vitro de extratos etanólicos da cúrcuma em pó, da curcumina disponível no comércio, dos três pigmentos curcuminóides purificados e do óleo essencial sobre algumas bactérias e fungos de relevância na indústria alimentícia.

\section{MATERIAL E MÉTODOS}

Rizomas de cúrcuma (Curcuma longa L., Zingiberaceae) fornecidos pela Universidade Federal de Lavras, foram lavados, selecionados, branqueados por 10 min, desidratados a $60^{\circ} \mathrm{C}$, triturados e peneirados em tamis de 40 mesh (BAMBIRRA et al., 2002). Para obtenção do extrato etanólico da cúrcuma em pó, $50 \mathrm{~g}$ da amostra foi submetida a refluxo em $500 \mathrm{~mL}$ de etanol, sob proteção da luz, por 90 minutos. A amostra foi filtrada, o solvente evaporado a $60^{\circ} \mathrm{C}$ (evaporador rotatório, Bücchi, Suíça) e o pó utilizado no preparo de solução de $5 \mathrm{mg} / \mathrm{mL}$ em etanol. A curcumina comercial $(50 \mathrm{mg})$ foi dissolvida em $100 \mathrm{~mL}$ de etanol, em banho ultrasônico, por 15 minutos. Os pigmentos curcuminóides purificados -curcumina, desmetoxicurcumina e bisdesmetoxicurcumina- foram obtidos por recristalização e cromatografia em coluna (PÉRET-ALMEIDA et al., 2005). Soluções etanólicas de cada pigmento foram preparadas na concentração de 5 $\mathrm{mg} / \mathrm{mL}$ cada. O óleo essencial foi obtido por hidrodestilação em aparelho de Clevenger $(60 \mathrm{~g}$ de cúrcuma em pó, $500 \mathrm{~mL}$ de água em balão de $1000 \mathrm{~mL}$ ) por $6 \mathrm{~h}$ (SILVA et al., 2005). Este foi utilizado sem diluições.

\section{Determinação dos pigmentos curcuminóides}

Os teores de pigmentos curcuminóides foram determinados por cromatografia líquida de alta eficiência<smiles></smiles><smiles>[R2]c1cc(C(=C)C)ccc1O</smiles>

\begin{tabular}{lll}
\hline Composto & $\mathrm{R} 1$ & $\mathrm{R} 2$ \\
\hline Curcumina & $\mathrm{OMe}$ & $\mathrm{OMe}$ \\
Desmetoxicurcumina & $\mathrm{H}$ & $\mathrm{OMe}$ \\
Bisdesmetoxicurcumina & $\mathrm{H}$ & $\mathrm{H}$ \\
\hline
\end{tabular}

Figura 1 - Estrutura química dos pigmentos curcuminóides da Curcuma longa L. 
(CLAE), em equipamento da Shimadzu (Kyoto, Japão), com detector arranjo de diodos a $425 \mathrm{~nm}$, coluna Shim-pack CLC$\mathrm{NH}_{2}$ (4,6 x 150 mm, Shimadzu, Kyoto, Japão), e fase móvel etanol:água $(85: 15, \mathrm{v} / \mathrm{v})$ a $1 \mathrm{~mL} / \mathrm{min}$. A identificação foi feita por comparação do tempo de retenção dos picos e confirmada pelo espectro de absorção no UV-visível. A quantificação foi feita usando as respectivas absortividades (PÉRET-ALMEIDA et al., 2005).

\section{Caracterização do óleo essencial da cúrcuma}

O rendimento do óleo essencial da cúrcuma foi determinado em função do volume obtido por massa de cúrcuma em pó usada. O óleo foi analisado quanto ao índice de refração a $30{ }^{\circ} \mathrm{C}$ (refratômetro de Abbé) e densidade a $30{ }^{\circ} \mathrm{C}$ (picnômetro). As substâncias voláteis foram obtidas por microextração em fase sólida, em fibra de $100 \mu \mathrm{m}$, de polidimetilsiloxano (57300-U, Supelco, PA, EUA) em contato com o headspace de $15 \mathrm{mg}$ da amostra aquecida a $80^{\circ} \mathrm{C} / 5 \mathrm{~min}$ (MATA et al., 2004). O cromatógrafo gasoso (CG) utilizado foi o GC-17A (Shimadzu, Kyoto, Japão), com detector de ionização de chama (FID), coluna capilar DB-1 (25 m x 0,23 mm; 0,52 $\mu \mathrm{m}$ ) e hélio como gás de arraste. A temperatura do injetor foi $220^{\circ} \mathrm{C}$ e a do detector $250{ }^{\circ} \mathrm{C}$. O tempo de dessorção foi 10 minutos e a temperatura, durante a corrida, variou de 60 a $200{ }^{\circ} \mathrm{C}$. Foram utilizados como padrão, borneol, limoneno e ar-turmerona.

\section{Determinação da atividade antimicrobiana in vitro}

Para a determinação da atividade antimicrobiana foram utilizadas culturas ATCC (Tabela 1). Antibióticos foram usados como controles positivos: para bactérias o cloranfenicol (Sigma, St. Louis, MO, EUA) e para leveduras e fungo a anfotericina B (Fungizon ${ }^{\circledR}$ Squibb, São Paulo, $\mathrm{SP}$, Brasil). Foram feitas soluções estoque aquosas com 1 $\mathrm{mg} / \mathrm{mL}$. Desta solução foram pipetados 5 e $10 \mu \mathrm{L}$, correspondentes a 5 e $10 \mu \mathrm{g}$ por disco, para cada antibiótico.

\section{Preparo dos inóculos}

As culturas padrão de bactérias e fungos foram mantidas sob refrigeração a $5{ }^{\circ} \mathrm{C}$. Utilizou-se para bactérias, repiques de 24 horas a $36 \pm 1{ }^{\circ} \mathrm{C}$ em ágar para antibióticos $\mathrm{n}^{\circ} .1$ e para as leveduras e fungo, repiques de 24 e 72 horas, respectivamente, em ágar Saboraud-Dextrose a $25 \pm 1{ }^{\circ} \mathrm{C}$ (Dialab Diagnósticos, Montes Claros, MG).

Uma suspensão padrão de microrganismo foi obtida transferindo colônias do meio inclinado para tubos de ensaio contendo $10 \mathrm{~mL}$ de solução salina peptonada estéril $(0,9 \%, \mathrm{p} / \mathrm{v})$ até obter, $50 \pm 1 \%$ de transmitância a $580 \mathrm{~nm}$, utilizando como branco a solução salina. No preparo do inóculo de Aspergillus niger adicionou-se à salina $0,1 \%$ v/v de Tween 80 para melhor dispersão das células. O branco foi adicionado de Tween 80, na mesma proporção.

A atividade antimicrobiana in vitro foi determinada pelo método de difusão em ágar (NUNAN et al., 1985). Discos de papel estéreis de $6 \mathrm{~mm}$ de diâmetro (Cecon, São Paulo, SP, Brasil) foram impregnados com 10 e $20 \mu \mathrm{L}$ dos extratos dos pigmentos e levados à estufa a $36^{\circ} \mathrm{C} / 24 \mathrm{~h}$, para a evaporação do solvente usado na solubilização dos extratos. Discos impregnados com os solventes e com 5 e $10 \mathrm{~mL}$ de antibióticos foram usados como controle.

Adicionou-se às placas de Petri, $20 \mathrm{~mL}$ de meio base e, após sua solidificação, $5 \mathrm{~mL}$ do meio de superfície com os microrganismos (inóculo de $0,05 \% \mathrm{v} / \mathrm{v}$, obtido adicionando-se uma alíquota da suspensão padrão ao meio ágar para antibióticos $n^{\circ} 1$ ). Os discos impregnados foram depositados sobre os meios de cultura e as placas incubadas a $36 \pm 1{ }^{\circ} \mathrm{C} / 24$ h e a $25 \pm 1{ }^{\circ} \mathrm{C} / 48 \mathrm{~h}$ para bactérias e fungos, respectivamente. Os diâmetros dos halos de inibição foram medidos com paquímetro.

\section{Análise estatística}

Os experimentos microbiológicos foram realizados com seis repetições e os químicos com três. Os resultados

Tabela 1 - Microrganismos utilizados nos ensaios de atividade antimicrobiana.

\begin{tabular}{lcc}
\hline \multicolumn{1}{c}{ Microrganismo } & Espécie & ATCC $^{1}$ \\
\hline Bactérias & Staphylococcus aureus & 6538 \\
& Bacillus subtilis & 6633 \\
& Salmonella choleraesuis & 14028 \\
& Escherichia coli & 8739 \\
\hline Fungos & Aspergillus niger & 16404 \\
& Saccharomyces cerevisae & 2601 \\
\hline
\end{tabular}

\footnotetext{
${ }^{1} \mathrm{ATCC}=$ American Type Culture Collection.
} 
foram submetidos à análise de variância e as médias comparadas pelo teste de Duncan, a 5\% de probabilidade.

\section{RESULTADOS E DISCUSSÃO}

Teores de pigmentos curcuminóides nos extratos da cúrcuma e no óleo essencial

Foram encontrados na cúrcuma em pó $4,1 \mathrm{~g} / 100 \mathrm{~g}$ de pigmentos curcuminóides (Tabela 2). Os três pigmentos estavam presentes, sendo a curcumina predominante (59\%), seguida da desmetoxicurcumina (29\%) e da bisdesmetoxicurcumina (12\%). Resultados semelhantes foram relatados para diferentes variedades de cúrcuma (GOVINDARAJAN, 1980; JAYAPRAKASHA et al., 2002). A curcumina disponível comercialmente, continha a mistura dos três pigmentos na mesma ordem de predominância, porém, os percentuais foram diferentes: $71 \%, 25 \%$ e $4 \%$, respectivamente. Este perfil de pigmentos curcuminóides já havia sido constatado por Péret-Almeida et al. (2005). Os três pigmentos curcuminóides, isolados, tiveram pureza confirmada por CLAE (Tabela 2).

\section{Caracterização do óleo essencial da cúrcuma}

O óleo essencial apresentou coloração amarela clara e odor forte e pungente característico. Obteve-se um rendimento de 4,4\% de óleo com densidade de $0,9068 \mathrm{~g} /$ $\mathrm{mL}$ e índice de refração de 1,5067 a $30^{\circ} \mathrm{C}$. Esses valores são similares aos descritos na literatura (BRAGA et al., 2003; GOVINDARAJAN, 1980; SILVA et al., 2005). O perfil cromatográfico do óleo essencial da cúrcuma por CG-FID (Figura 2) indicou a presença de picos com tempos de retenção que variaram de 5 a 80 minutos, incluindo limoneno, borneol e ar-turmerona (picos 1, 2 e 3, respectivamente), sendo o último o predominante $(\sim 58 \%)$. As estruturas químicas destes compostos estão apresentadas na Figura 3.

Tabela 2 - Teores de pigmentos curcuminóides nos diferentes produtos da cúrcuma.

\begin{tabular}{lccc}
\hline \multicolumn{1}{c}{ Amostra } & \multicolumn{3}{c}{ Teores de pigmentos $(\mathrm{g} / \mathrm{g})$} \\
\cline { 2 - 4 } & Curcumina & DMC & BDMC \\
\hline Cúrcuma em pó & $0,024 \pm 0,001$ & $0,012 \pm 0,001$ & $0,005 \pm 0,001$ \\
Curcumina comercial & $0,713 \pm 0,06$ & $0,246 \pm 0,05$ & $0,041 \pm 0,02$ \\
Curcumina isolada & $1,00 \pm 0,02$ & nd & nd \\
Desmetoxicurcumina isolada & nd & $1,00 \pm 0,02$ & nd \\
Bisdesmetoxicurcumina isolada & nd & nd & $1,00 \pm 0,01$ \\
Óleo essencial & $0,002 \pm 0,000$ & $0,0006 \pm 0,0001$ & nd \\
\hline
\end{tabular}

$\mathrm{DMC}=$ Desmetoxicurcumina; $\mathrm{BDMC}=$ Bisdesmetoxicurcumina .

nd - não detectado (limite de detecção em $\mathrm{g} / \mathrm{g}=2,7 \times 10^{-5}$ para curcumina, $1,2 \times 10^{-5}$ para DMC e $0,57 \times 10^{-5}$ para BDMC).

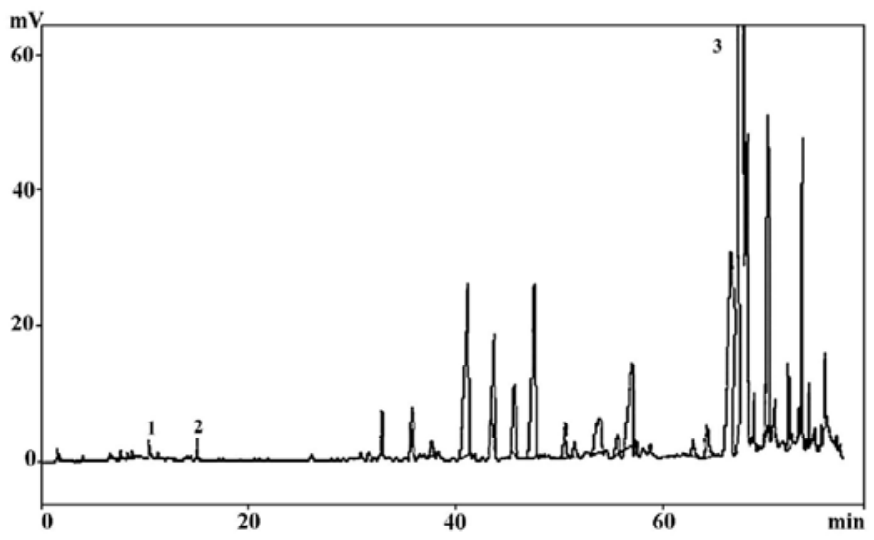

Figura 2 - Perfil cromatográfico das substâncias voláteis dos óleos essenciais da cúrcuma obtidos por hidrodestilação em aparato de Clevenger.

Condições cromatográficas: CG-IC, coluna capilar DB-1 $(25 \mathrm{~m} \times 0,32 \mathrm{~mm} ; 0,52 \mu \mathrm{m})$, gás de arraste hélio; temperaturas do injetor 220 ${ }^{\circ} \mathrm{C}$; do detector $250{ }^{\circ} \mathrm{C}$, e da coluna: programação de 60 a $200{ }^{\circ} \mathrm{C}$. Picos 1,2 e 3, foram identificados como limoneno, borneol e arturmerona respectivamente. 
<smiles>C=C(C)C1CC=C(C)CC1</smiles>

Limoneno $\left(\mathrm{C}_{10} \mathrm{H}_{16}\right)$<smiles>CC12CCC(CC1)C2O</smiles>

Borneol $\left(\mathrm{C}_{10} \mathrm{H}_{18} \mathrm{O}\right)$<smiles>CC(C)=CC(=O)CC(C)c1ccc(C)cc1</smiles>

ar-Turmerona

$\left(\mathrm{C}_{15} \mathrm{H}_{20} \mathrm{O}\right)$

Figura 3 - Estruturas químicas das substâncias do óleo essencial da Cúrcuma longa L. identificadas por CG-FID.

De acordo com Govindarajan (1980) e Singh et al. (2002) são vários os componentes voláteis do óleo essencial da cúrcuma, dentre eles, mono e sesquiterpenos alifáticos ou oxigenados. Braga et al. (2003) e Mata et al. (2004) reportaram também a presença de pineno, sabineno, 1,8cineol, 1,4-terpineol, carofileno, curcumeno, bisaboleno, felandreno, atlantone, zingibereno, turmerona e arturmerona, em produtos de cúrcuma produzida no Brasil. Essas substâncias estão, possivelmente, presentes na amostra analisada; entretanto, os respectivos padrões não estavam disponíveis para identificação. Além disso, essas contribuem com um pequeno porcentual no perfil cromatográfico, sendo a ar-turmerona o componente predominante.

\section{Atividade antimicrobiana in vitro da cúrcuma e dos pigmentos curcuminóides}

Não foi observada atividade antimicrobiana dos extratos etanólicos da cúrcuma em pó e da curcumina comercial para os microrganismos testados. Com relação aos pigmentos purificados, a curcumina e a desmetoxicurcumina em concentrações de até $10 \mu \mathrm{g} / \mu \mathrm{L}$ não apresentaram atividade antimicrobiana. Entretanto, a bisdesmetoxicurcumina apresentou atividade inibitória para o Bacillus subtilis na concentração de $10 \mu \mathrm{g} / \mu \mathrm{L}$.

Os resultados obtidos para extratos brutos, contendo uma mistura dos pigmentos curcuminóides confirmam achados de Singh et al. (2002) e Tönnesen \& Karlsen (1987) para E. coli, Salmonella, S. aureus e $S$. epidermidis e por Apisariyakul et al. (1995) para fungos patogênicos e leveduras. De acordo com Tönnesen \& Karlsen (1987), a atividade antimicrobiana pode ser detectada apenas em concentrações elevadas ou em menores concentrações, quando expostas à radiação no visível.

A atividade antimicrobiana dos pigmentos purificados foi descrita pela primeira vez. Os halos para a bisdesmetoxicurcumina $(10 \mathrm{mg} / \mathrm{mL} ; 20 \mathrm{~mL} /$ disco $)$ foram maiores $(46 \%)$ que os halos para o cloranfenicol $(1 \mathrm{mg} / \mathrm{mL}$;
$10 \mathrm{~mL} /$ disco), indicando que este pigmento apresentou atividade antimicrobiana significativa para o B. subtilis. Estes resultados indicam um potencial uso da bisdesmetoxicurcumina na inibição do $B$. subtilis.

\section{Atividade antimicrobiana in vitro do óleo essencial da cúrcuma}

Não foi observada atividade inibitória do óleo essencial para $S$. aureus e para $C$. albicans. O óleo essencial da cúrcuma (Tabela 3 ) inibiu o desenvolvimento de B. subtilis, S. choleraesuis, E. coli, A. niger e $S$. cerevisiae. Resultados similares foram obtidos por Negi et al. (1999) que verificaram a inibição do crescimento de $E$. coli e B. subtilis com o óleo essencial. Singh et al. (2002) observaram atividade inibitória para o $S$. aureus, entretanto, usaram extratos em etileno glicol, contendo $2000 \mu \mathrm{g}$ do óleo/disco.

Maior atividade foi observada contra o B. subtilis, seguida do $S$. choleraesuis nas duas concentrações empregadas. A maior atividade observada para bactérias Gram positivas também foi relatada por Singh et al. (2002). De acordo com Gul et al. (2004), este resultado pode estar associado ao fato dessas bactérias não possuírem a membrana externa capaz de restringir a penetração de substâncias exógenas.

Ao aumentar a concentração do óleo essencial de 45 para $90 \mu \mathrm{g} /$ disco, os diâmetros dos halos aumentaram, com inibição de $60 \%$ para E. coli e $131 \%$ para B. subtilis. Halos maiores foram observados para o óleo essencial $(90$ $\mu \mathrm{g} /$ disco), comparados aos dos antibióticos (10 $\mu \mathrm{g} /$ disco), exceto para a $E$. coli. Os halos de inibição formados com o óleo essencial aumentaram $6 \%$ para $S$. choleraesuis e $A$. niger; e de $44 \%$ para o B. subtilis, em relação aos halos obtidos com os antibióticos.

Os resultados indicaram que o óleo essencial da cúrcuma constitui um potencial agente antimicrobiano. Vários componentes poderiam ser responsáveis por essa atividade como fenóis, terpenos, aldeídos e cetonas, sendo que vários destes já foram identificados no óleo essencial 
Tabela 3 - Atividade antimicrobiana in vitro do óleo essencial da cúrcuma.

\begin{tabular}{|c|c|c|c|c|}
\hline \multirow{3}{*}{ Microrganismo } & \multicolumn{4}{|c|}{ Diâmetro do halo de inibição $(\mathrm{mm}) /$ concentração } \\
\hline & \multicolumn{2}{|c|}{ Óleo essencial da cúrcuma } & \multicolumn{2}{|c|}{ Antibiótico } \\
\hline & $45 \mu \mathrm{g} / \mathrm{disco}$ & $90 \mu \mathrm{g} /$ disco & $5 \mu \mathrm{g} / \mathrm{disco}$ & $10 \mu \mathrm{g} / \mathrm{disco}$ \\
\hline B. subtilis & $8,02 \pm 0,19^{\mathrm{a}, \mathrm{z}}$ & $18,51 \pm 0,49^{\mathrm{a}, \mathrm{x}}$ & $12,71 \pm 0,34^{\text {a,y }}$ & $12,87 \pm 0,45^{\mathrm{a}, \mathrm{y}}$ \\
\hline S. choleraesuis & $7,33 \pm 0,20^{\mathrm{b}, \mathrm{z}}$ & $13,66 \pm 0,30^{\mathrm{b}, \mathrm{x}}$ & $12,81 \pm 0,36^{\mathrm{a}, \mathrm{y}}$ & $12,90 \pm 0,32^{\mathrm{a}, \mathrm{y}}$ \\
\hline E. coli & $6,30 \pm 0,17^{\mathrm{c}, \mathrm{y}}$ & $10,09 \pm 0,46^{\mathrm{d}, \mathrm{x}}$ & $10,03 \pm 0,19^{\mathrm{c}, \mathrm{x}}$ & $10,20 \pm 0,15^{\mathrm{c}, \mathrm{x}}$ \\
\hline A. niger & $6,76 \pm 0,38^{\mathrm{bc}, \mathrm{z}}$ & $12,02 \pm 0,47^{\mathrm{c}, \mathrm{x}}$ & $11,21 \pm 0,42^{b, y}$ & $11,38 \pm 0,37^{\text {by }}$ \\
\hline S. cerevisae & $6,18 \pm 0,06^{\mathrm{c}, \mathrm{z}}$ & $11,55 \pm 0,41^{\mathrm{c}, \mathrm{x}}$ & $10,08 \pm 0,23^{\mathrm{c}, \mathrm{y}}$ & $10,10 \pm 0,41^{\mathrm{c}, \mathrm{y}}$ \\
\hline
\end{tabular}

${ }^{1}$ Valores médios ( \pm desvios-padrão) com letras diferentes (x-z) na mesma linha e (a-e) na mesma coluna são diferentes (teste Duncan, $\mathrm{p}<0,05)$. O diâmetro do halo corresponde à medida total incluindo a medida do halo de papel.

da cúrcuma (BRAGA et al., 2003; MATA et al., 2004). De acordo com Lee et al. (2003) e Negi et al. (1999), a fração do óleo essencial da cúrcuma com maior percentual de arturmerona foi a que apresentou maior atividade antimicrobiana. Entretanto, estudos são necessários para identificar quais seriam os compostos responsáveis pela atividade biocida ou bioestática no óleo essencial da cúrcuma e as possíveis interações.

\section{CONCLUSÕES}

Não foi observada atividade dos extratos etanólicos da cúrcuma, da curcumina comercial e dos pigmentos purificados curcumina e desmetoxicurcumina para S. aureus, B. subtilis, S. choleraesuis, E. coli, A. niger, S. cerevisiae, e C. albicans; apenas o extrato etanólico da bisdesmetoxicurcumina apresentou atividade antimicrobiana in vitro para o Bacillus subtilis.

O óleo essencial da cúrcuma obtido por hidrodestilação em aparelho de Clevenger apresentou atividade antimicrobiana in vitro para o B. subtilis, $S$. choleraesuis, E. coli, A. niger $S$. cerevisiae. Essa atividade aumentou com o aumento da concentração.

Os resultados indicaram ser a bisdesmetoxicurcumina e o óleo essencial da cúrcuma, principalmente o último, antimicrobianos em potencial para uso na indústria alimentícia.

\section{AGRADECIMENTOS}

Ao Departamento de Agricultura da UFLA pelos rizomas da cúrcuma. À Profa. Marilia Martins Melo da Escola de Veterinária da UFMG pela doação de arturmerona. À FAPEMIG pelo apoio financeiro ao projeto CAG-428/03.

\section{REFERÊNCIAS BIBLIOGRÁFICAS}

APISARIYAKUL，A.; VANITTANAKOM， N.; BUDDHASUKH, D. Antifungal activity of turmeric oil extracted from Curcuma longa (Zingiberaceae). Journal of Ethnopharmacology, Limerick, v. 49, p. 163-169, 1995.

BAMBIRRA, M. L. A.; JUNQUEIRA, R. G.; GLORIA, M. B. A Influence of post harvest processing conditions on yield and quality of ground turmeric (Curcuma longa L.). Brazilian Archives of Biology and Technology, Curitiba, v. 45, n. 4, p. 423-429, 2002.

BRAGA, M. E. M.; LEAL, P. F.; CARVALHO, J. E.; MEIRELES, M. A. A. Comparison of yield, composition and antioxidant activity of turmeric (Curcuma longa L.) extracts obtained using various techniques. Journal of Agricultural and Food Chemistry, Easton, v. 51, p. 66046611, 2003.

GOVINDARAJAN, V. S. Turmeric: chemistry, technology and quality. Critical Reviews in Food Science and Nutrition, Boca Raton, v. 12, n. 3, p. 199-301, 1980.

GUL, N.; MUJAHID, T. Y.; JEHAN, N.; AHMAD, S. Studies on the antibacterial effect of different fractions of Curcuma longa against urinary tract infection isolates. Pakistan Journal of Biological Sciences, v. 7, n. 12, p. 2055-2060, 2004.

JAYAPRAKASHA, G. K.; RAO, L. J. M.; SAKARIAH, K. $\mathrm{S}$. Improved HPLC method for the determination of curcumin, demethoxycurcumin and bisdemethoxycurcumin. Journal of Agricultural and Food Chemistry, Easton, v. 50, p. 3668-3672, 2002. 
KIM, M. K.; CHOI, G. J.; LEE, H. S. Fungicidal property of Curcuma longa L. rhizome-derived curcumin against phytopathgenic fungi in a greenhouse. Journal of Agricultural and Food Chemistry, Easton, v. 51, p. 15781581, 2003.

LEE, H. S.; CHOI, K. J.; CHO, K. Y.; AHN, Y. J. Fungicidal activity of ar-turmerone identified in Curcuma longa rhizome against six phytopathogenic fungi. Agricultural and Chemical Biotechnology, [S.1.], v. 46, n. 1, p. 25-28, 2003.

MATA, A. R.; NELSON, D. L.; AFONSO, R. J. C. F.; GLÓRIA, M. B. A.; JUNQUEIRA, R. G. Identificação de compostos voláteis da cúrcuma empregando microextração por fase sólida e cromatografia gasosa acoplada à espectrometria de massas. Ciência e Tecnologia de Alimentos, Campinas, v. 24, n. 1, p. 151-157, 2004.

NEGI, P. S.; JAYAPRAKASHA, L.; RAO, J. M.; SAKARIAH, K. K. Antibacterial activity of turmeric oil: a byproduct from curcumin manufacture. Journal of Agricultural and Food Chemistry, Easton, v. 47, p. 4297 4300, 1999.

NUNAN, E. A.; CAMPOS, L. M. M.; PAIVA, R. L. R.; OLIVEIRA, S. T.; DADOUN, H. A.; OLIVEIRA, A. B. Estudo da atividade antimicrobiana de extrato de folhas de Aristolochia gigantea Mart e Zucc. Revista de Farmácia e Bioquímica, Belo Horizonte, v. 6, p. 33 -40, 1985.

PÉRET-ALMEIDA, L.; CHERUBINO, A. P. F.; ALVES, R. J.; GLÓRIA, M. B. A. Separation and determination of the physico-chemical characteristics of curcumin, demethoxycurcumin and bisdemethoxycurcumin. Food Research International, Barking, v. 38, p. 1039-1044, 2005.

SILVA, L. V.; NELSON, D. L.; DRUMOND, M. F. B.; DUFOSSE, L.; GLORIA, M. B. A. Comparison of hydrodistillation methods for the deodorization of turmeric. Food Research International, Barking, v. 38, p. 1087-1096, 2005.

SINGH, R.; CHANDRA, R.; BOSE, M.; LUTHRA, P. M. Antibacterial activity of Curcuma longa rhizome extract on pathogenic bacteria. Current Science, Bangalore, v. 83 , n. 6, p. 737-740, 2002.

TÖNNESEN, H. H.; KARLSEN, J. Studies on curcumin and curcuminoids IX: investigation of the photobiological activity of curcumin using bacterial indicator systems. Journal of Pharmaceutical Science, Washington, v. 76, p. 373-373, 1987. 